\title{
Improved Algorithms for Reservoir Capacity Calculation Incorporating Storage-Dependent Losses and Reliability Norm
}

\author{
SHARAD M. LeLE ${ }^{1}$ \\ Department of Computer Science and Automation, Indian Institute of Science, Bangalore, India
}

\begin{abstract}
Two algorithms that improve upon the sequent-peak procedure for reservoir capacity calculation are presented. The first incorporates storage-dependent losses (like evaporation losses) exactly as the standard linear programming formulation does. The second extends the first so as to enable designing with less than maximum reliability even when allowable shortfall in any failure year is also specified. Together, the algorithms provide a more accurate, flexible and yet fast method of calculating the storage capacity requirement in preliminary screening and optimization models.
\end{abstract}

\section{INTRODUCTION}

The sequent-peak procedure is a well-known simplistic algorithm for determining the storage capacity requirement for a reservoir [Thomas and Fiering, 1963]. It has also been extended to cascaded multireservoir systems in analyses based on a dynamic programming approach [Murray and Weissbeck, 1980]. But the procedure suffers from serious limitations:

1. Storage-dependent losses or releases cannot be included in the calculations.

2. It cannot be used for complex multireservoir systems.

3. It cannot be used when less than maximum reliability (percentage of periods in which target demand is met) is desired; specification of the shortfall that may be allowed in any failure year is, therefore, out of the question.

4. It cannot be used when the reservoir operating policy is not the standard one. (A standard operating policy (SOP) is one under which the seasonal release is equal to the target release or all the available water, whichever is less.)

On the other hand, the procedure has the significant advantage of not being limited by the number of years and seasons of inflows used in the analysis, a factor that is crucial in determining practical solvability in analyses based on the commonly used linear programming (LP) formulation [Loucks et al., 1981, pp. 236-238]. Further (at least in the case of single or cascaded multireservoir systems), it enables the analyst to tackle problems with significantly nonlinear objective functions with the help of direct-search optimization methods.

Some attempts have been made to overcome the shortcomings mentioned above. Modifications have been easily made to enable calculation of capacity for different levels of reliability [Loucks, 1976]. Tejada-Guibert [1978] has presented an iterative algorithm for calculating storage capacity at maximum reliability in which evaporative losses are accurately included and also a half-interval search procedure for determining the storage required for a desired reliability. None of these algorithms, however, is able to provide for specification of the extent of shortfall that may be allowed in any failure or "short" year.

\footnotetext{
${ }^{1}$ Now at Energy and Resources Group, University of California, Berkeley.

Copyright 1987 by the American Geophysical Union.
}

Paper number 7 W0617.

0043-1397/87/007W-0617\$05.00
In this paper we present two simple algorithms that overcome limitations (1) and (3) completely. In part I we describe an algorithm that is based on the sequent-peak procedure but takes into consideration storage-dependent losses as "exactly" as they are included in the standard LP formulation. (Although they are called "evaporation losses" hereinafter, it should be noted that they could be any kind of storagedependent losses or withdrawals.) In part II we show how this improved algorithm may be used in a procedure that would enable one to calculate the active storage capacity requirement when the reliability norm specifies not only the maximum number of failure years but also the allowable shortfall in any failure year. Finally, we indicate how the procedure may be able to overcome limitations (2) and (4) to some extent.

\section{Part I: AN Algorithm for InCORporating STORAGE-DEPENDENT LOSSES INTO THE Sequent-Peak Procedure}

We begin by defining the notation used. The original sequent-peak procedure is outlined in section 2.1 , and the algorithm is then derived from it in the subsequent sections.

a slope of the area-capacity curve for the reservoir, $L^{-1}$

$b$ reservoir water spread at dead storage level, $L^{2}$;

$A_{t}$ reservoir water spread at the beginning of time period $t, L^{2}$;

$C U_{t}$ fixed loss from the reservoir in time period $t$ (such as local consumptive use), $\boldsymbol{L}^{3}$;

$e_{t}$ average rate of evaporation from reservoir surface in period $t, L$;

$E V_{t}$ volume of water lost owing to evaporation in period $t, L^{3}$;

ICRIT last period in critical sequence of inflows;

IOVF last period in which the reservoir spilled over before encountering the critical sequence of inflows;

$K_{a}$ approximate active storage capacity of reservoir calculated from sequent-peak procedure ignoring storage-dependent losses, $L^{3}$;

$K_{a}{ }^{*}$ exact active storage capacity, $L^{3}$;

$K$, sequential cumulative deficit at the end of period $t, L^{3}$;

$Q_{t}$ inflow into reservoir during period $t, L^{3}$;

$R_{t}$ release from reservoir during period $t, L^{3}$; 
$S_{t}$ active storage in the reservoir at the beginning of period $t, L^{3}$;

$Y$ total number of years in the inflow sequence;

$S$ number of within-year seasons that each year is divided into;

$T$ total number of periods in the inflow sequence $(=Y S)$.

Note that it is assumed as usual that if $t=T$ then $t+1=1$, i.e., the given set of inflows repeats itself. In other words, the sequence of inflows may be looked upon as not a "straight line" but a "closed circle." Therefore a phrase such as "backward from $t=t_{1}$ to $t=t_{2}$ " means (when $t_{2}>t_{1}$ ) "from $t=t_{1}$ down to $t=1$ and then $t=T$ down to $t=t_{2}$." Similarly, "forward from $t=t_{1}$ to $t=t_{2}$ " means (when $t_{2}<t_{1}$ ) "up to $t=T$ and then from $t=1$ up to $t=t_{2}$."

\subsection{Sequent-Peak Procedure}

Given the values of the inflows $Q_{t}$, releases $R_{t}$, and fixed losses $C U_{t}$ (and assuming $E V_{t}=0$ ) for $t=1, \cdots, T$, the minimum active storage requirement $K_{a}$ can be calculated using the sequent-peak procedure as follows.

Step 1 . Set $K_{0}=0$.

Step 2. For $t=1$ to $T$, calculate $K_{t}=\max \left\{0,\left(K_{t-1}+R_{t}\right.\right.$ $\left.\left.+C U_{t}-Q_{t}\right)\right\}$.

Step 3. If $K_{T}=K_{0}$, then go to step 4; else if this is the first iteration, then set $K_{0}=K_{T}$ and go to step 2; else STOP: sequent-peak analysis failed because gross utilization is greater than the average inflow.

Step 4. $K_{a}=$ maximum $\left\{K_{t}\right\}$ over $t=1, \cdots, T$.

Note that $R_{1}$ and $C U_{t}$ values are actually given/specified only for $t=1$ to $S$; since they repeat from year to year, $R_{t}=$ $R_{t-s}$ for $t>S$, etc.

Evaporation loss in any period $t$ is actually proportional to the average reservoir water spread area during that period, which in turn depends on the storage levels in the reservoir at the beginning and the end of that period, i.e., $S_{t}$ and $S_{t+1}$. Since it is not possible to determine $S_{t}$ without knowing $K_{a}$, the above procedure obviously cannot include any such storage-dependent losses.

\subsection{Inclusion of Evaporation Losses}

The manner in which evaporation losses are incorporated in the standard LP formulation provides a pointer to the modifications required in the sequent-peak procedure. In the LP formulation, the area-capacity relationship for the reservoir is approximated as

$$
A_{t}=a S_{t}+b
$$

(Note that this linearization of the area-storage relationship is an approximation that may lead to distortions in some cases, but it is nevertheless used very commonly. Our algorithm uses the same approximation and so is "exact" only to the extent that the LP formulation is.)

The evaporation loss in any period $t$ is taken to be the product of the average reservoir water spread during period $t$ and the average evaporation rate for that period. Hence

$$
E V_{t}=e_{t}\left[\left(A_{t}+A_{t+1}\right) / 2\right]
$$

Using (1) and (2), one obtains an expression for the evaporation loss as a function of the active storage volumes:

$$
E V_{t}=e_{t}\left[a\left(S_{t}+S_{t+1}\right) / 2+b\right]=S_{t}\left(a e_{t} / 2\right)+S_{t+1}\left(a e_{t} / 2\right)+e_{t} b
$$

Now, the original storage-continuity relationship in the standard LP model [Loucks et al., 1981, pp. 343-348] is

$$
S_{t}+Q_{1}-R_{t}-C U_{t}-E V_{t} \geq S_{t+1} \quad t=1, \cdots, T
$$

which, using (3), can now be rewritten as

$$
S_{t}\left(1-e_{t} a / 2\right)+Q_{t}-R_{t}-C U_{t}-e_{t} b \geq S_{t+1}\left(1+e_{t} a / 2\right)
$$

or

$$
S_{t+1} \leq\left[S_{\imath}\left(1-e_{t} a / 2\right)+Q_{t}-R_{t}-C U_{t}-e_{t} b\right] /\left(1+e_{t} a / 2\right)
$$

The other set of constraints in the LP model that goes along with (4) is the set of constraints specifying the minimum active storage capacity:

$$
K_{a}^{*} \geq S_{t} \quad t=1, \cdots, T
$$

(The symbol $K_{a}{ }^{*}$ is used instead of $K_{a}$ to indicate that this would be the exact value of capacity required and not the approximate one given by the sequent-peak procedure.) This constraint, when combined with (4), gives a rule for determining $S_{t+1}$, given $S_{t}$ and $K_{a}^{*}$, which is

$$
\begin{aligned}
S_{t+\mathrm{t}}= & \min \left\{K_{a}^{*},\right. \\
& \left.\cdot\left[\left(S_{\mathrm{t}}\left(1-e_{\mathrm{t}} a / 2\right)+Q_{\mathrm{t}}-R_{\mathrm{t}}-C U_{\mathrm{t}}-e_{\mathrm{t}} b\right) /\left(1+e_{\mathrm{t}} a / 2\right)\right]\right\}
\end{aligned}
$$

Note that if $S_{t+1}$ is equal to $K_{a}^{*}$, it indicates that the difference between the second term on the right-hand side and $K_{a}{ }^{*}$ is the volume of water that has spilled over.

The question of course is "how can the values of $S_{t}$ and $K_{a}^{*}$ be known (when $K_{a}^{*}$ itself is the basic unknown to be determined)?". We shall now outline the algorithm that shows how this can be done systematically.

The starting point of the algorithm is the fact that if one is sure that during a certain period (or sequence of periods) there has been no spillover, then for only that period (or sequence of periods)

$$
S_{t+1}=\left[S_{t}\left(1-e_{t} a / 2\right)+Q_{t}-R_{t}-C U_{t}-e_{t} b\right] /\left(1+e_{t} a / 2\right)
$$

(which temporarily obviates the need to know $K_{a}{ }^{*}$ ). Now, there does exist such a sequence of periods within which one can be sure that there have been no spillovers, namely, the critical sequence of inflows. Further, one knows that (1) the active storage at the end of the sequence will be zero (the reservoir will be at its maximum draw-down level) and (2) it is possible to identify the beginning and end of this sequence from the calculations in the sequent-peak procedure.

The identification of the critical sequence is a simple matter. In step 4 of the sequent-peak procedure, all the values of the sequential cumulative deficit $K_{t}$ have to be examined. Clearly, the value of $t$ corresponding to the largest deficit $K_{t}$ (which is then taken as $K_{a}$ ) is nothing but the last critical period, say ICRIT. Hence from remark 1 above,

$$
S_{\text {ICRTT +1 }}=0
$$

Moreover, the beginning of the critical sequence is the last time the reservoir spilled over or "overflowed" before this lowinflow sequence was encountered, or going backward from $t=$ ICRIT, it would be the first period for which $K_{t}=0$. Let this period be IOVF. Note that the storage in the reservoir at the end of this period as indicated by the sequent-peak procedure (say, $S_{\mathrm{IOVF}+1}$ ) would be equal to $K_{\sigma}$. One could then 
rewrite (6) as

$$
S_{t}=\left[S_{t+1}\left(1+e_{t} a / 2\right)+R_{t}+C U_{t}+e_{t} b-Q_{t}\right] /\left(1-e_{t} a / 2\right)
$$

and calculate backward from $t=$ ICRIT to $t=$ IOVF +1 .

Note that $S_{\mathrm{IOvF+1}}$ is the storage volume that should have been there in the reservoir at the beginning of the critical sequence if the reservoir were to provide for all the releases, consumptive losses and exact evaporation losses in that sequence of periods. Hence in general, $S_{10 v F+1}$ would be greater than $S_{\mathrm{IOVF}+1}{ }^{\prime}\left(=K_{a}\right)$, and in fact, it is the desired exact active storage, i.e.,

$$
K_{a}^{*}=S_{\text {lOVF }+1}
$$

This claim, however, needs to be qualified. Since the critical sequence could be different for different levels of gross utilization, the inclusion of the evaporation losses may lead to a shift in the critical sequence itself. Such a shift would manifest itself in a negative value of $S_{t}$ (i.e., a deficit) in some period subsequent to ICRIT. To take care of this possibility, the remaining values of $S_{t}$ need to be calculated using rule (5) with $K_{a}^{*}$ from (8) above and checked for negativity. If any $S$, is found to be negative, the sequent-peak procedure would have to be repeated with the new (nonzero) values of evaporative losses $E V_{\text {, }}$ (obtained using the current values of $S_{t} s$ in (3)); ICRIT and IOVF would have to be recalculated and the procedure repeated all over again.

\subsection{Algorithm I}

Thus the algorithm may be stated succinctly as follows.

Step 1: Initialization.

1. Initialization of $Q_{t}, R_{b}, e_{t}, C U_{p}, a, b$.

2. $E V_{\mathrm{t}}=0$ for all $t=1, \cdots, T$.

Step 2: Sequent-Peak Procedure.

1. $K_{0}=0$.

2. Calculate $K_{t}=\max \left\{0,\left(K_{t-1}+R_{t}+C U_{t}+E V_{t}-Q_{t}\right)\right\}$ for all $t=1, \cdots, T$.

3. If $K_{T}=K_{0}$, then go to number 4; else if this is the first iteration in step 2, then put $K_{0}=K_{T}$ and go to number 2; else STOP: sequent-peak analysis failed because gross utilization is greater than the average inflow.

4. Find the period for which $K_{t}$ is the maximum of all $K_{t}$, $t=1, \cdots, T$; call this ICRIT.

5. Search backward from $t=$ ICRIT until $K_{t}=0$ is encountered for the first time. Assign to IOVF this value of $t$.

Step 3: Recalculation.

1. $S_{\text {ICRIT }+1}=0$.

2. For $t=\mathrm{ICRIT}+1$ backward to $t=\mathrm{IOVF}+1$, calculate $S_{t}=\left[S_{t+1}\left(1+e_{t} a / 2\right)+R_{t}+C U_{t}+e_{t} b-Q_{t}\right] /\left(1-e_{t} a / 2\right)$.

3. $K_{a}{ }^{*}=S_{\mathrm{IOVF}+1}$.

Step 4: Checking.

1. For $t=$ ICRIT +1 forward to $t=\operatorname{IOVF}-1$, calculate $S_{t+1}=\min \left\{K_{0}{ }^{*},\left[S_{t}\left(1-e_{t} a / 2\right)+Q_{t}-R_{t}-C U_{t}-e_{t} b\right] /\left(1+e_{t} a / 2\right)\right\}$.

2. If any $S_{t}$ is negative, then go to number 3; else STOP; $K_{a}{ }^{*}$ is the required exact active storage.

3. Calculate $E V_{t}=e_{t}\left[a\left(S_{t}+S_{t+1}\right) / 2+b\right]$ for all $t=1$, $\cdots, T$, and go back to step 2 .

3. Part II: Procedure to Calculate the Active Storage Capacity Requirement for DifFerent LeVels of Reliability With a SPecific Degree of Shortfall in ANy Failure Year

In this part a procedure is outlined which enables one to calculate the active storage capacity for less than maximum reliability when the allowable shortfall in any failure year is also specified.

\subsection{Further Notation and Definitions}

In addition to the symbols defined in section 2 , we define the following:

$f$ number of failure years allowed;

$d$ reliability (expressed as a fraction);

$\alpha$ fraction of normal yield desired in a failure year ( = 1 - maximum allowable fractional shortfall);

$R_{t}^{*} \quad$ target release in period $t\left[L^{3}\right]$.

Given the desired degree of reliability $d$, the allowable number of failure years can be calculated using the definition of reliability:

$$
d=(Y-f) /(Y+1)
$$

Therefore

$$
f=\operatorname{rnd}\{Y-(Y+1) d\}
$$

where rnd means rounded off. So, the actual set of releases should be $R_{t}=\alpha R_{t}^{*}$ for all the within-year periods in a failure year and $R_{t}=R_{t}^{*}$ for all other periods.

In the LP formulation that allows for less than maximum reliability, the identification of the actual failure years has to be done by trial and error, since the critical years are not known beforehand [Loucks et al., 1981, pp. 348-349]. Now, however, using the algorithm from part I, we can identify the critical years dynamically, i.e., during the calculation itself, and modify or "reset" the releases for those years to lower values.

\subsection{Procedure $I I$}

Step 1. Set $R_{t}=R_{t}^{*}$ for all $t=1, \cdots, T$.

Step 2. Execute "modified" algorithm (of part I of this paper) to determine $K_{a}{ }^{*}$ and ICRIT for current set of releases $\left\{R_{t}\right\}$.

Step 3. If this is the $(f+1)$ th iteration (i.e., if releases have been "reset" for $f$ years), then STOP; else go on to step 4.

Step 4. From ICRIT, determine the current critical year, say ICY. If ICY is the same as for the last iteration, then reset $R_{t}=\alpha R_{t}^{*}$ for $t$ corresponding to periods in year previous to ICY; else reset $R_{t}=\alpha R_{t}^{*}$ for $t$ corresponding to periods in the ICYth year. Go back to step 2.

Sometimes the critical period happens to be the first season of the "wet" year immediately following a drought sequence. In such a case it is customary to choose the last year of the drought sequence as the critical year rather than the wet year to which ICRIT belongs.

Thus it is possible to generate a complete and accurate "family" of storage-yield curves for a set of inflows of any length. Clearly, the above procedure could be modified easily so as to reset the releases for one season at a time rather than for all the seasons in a year at once. It can also be easily altered to suit the concept of providing secondary yields of less than maximum reliability (in addition to a firm yield with maximum reliability) as outlined by Loucks [1976, pp. 168169] or Loucks et al. [1981, pp. 350-351].

\section{Concluding Remarks}

The algorithm for incorporating storage-dependent losses was tested on a real 50-years and 12-months inflow sequence and was found to give the same results as those obtained by using the LP formulation to the last significant digit. The 
second procedure was also implemented as a computer program; it provided a very quick and flexible way of accurately determining the effect of changes in the reliability norm, including in the percentage shortfall allowed, on the storage capacity requirement.

Some remarks may be pertinent here as to the usefulness and scope of the algorithms. First, the inclusion of evaporation losses is itself of significance, because although the average annual evaporation loss may be only about 6-10\% of the storage capacity of the reservoir, the difference in the storage capacity requirement as calculated before and after the inclusion of evaporation losses is usually much higher, with the magnitude of the increase depending on the length of the critical sequence. (For example, this increase was $30 \%$ in the case of the same inflow data mentioned above.) Consequently, a reasonably accurate estimation of the evaporative losses is necessary for the accurate estimation of the active storage capacity requirement, especially at sites with high rates of evaporation.

Second, although we have developed the algorithms for the case when the reservoir operating policy is the SOP, they could be used (with minor modifications) under other operating policies, too. For instance, a very general $S$-type linear decision rule (LDR) of the kind

$$
R_{t}=c_{s} S_{t}+d_{s}
$$

(subscript $s$ denotes that the decision parameters are different only from season to season, not year to year) could be incorporated easily by substituting $\left(c+e_{t} a / 2\right)$ for $\left(e_{t} a / 2\right)$ and $\left(d+b e_{t}\right)$ for $\left(b e_{t}\right)$ in the statement of the algorithm in section 2.3. Similarly, it should be possible to incorporate an $S Q$-type LDR. Since LDRs have not been found to be very appropriate for reservoir capacity screening models [Stedinger, 1984], this may not be a very useful avenue for further exploration; nevertheless, it serves to demonstrate the inherent flexibility of the algorithms. A more useful extension would be one whereby a "less than $100 \%$ minimum storage reliability" could be incorporated; resetting $R_{t}$ to $\left(R_{t}^{*}-S_{\min }\right)$ rather than $\alpha R_{t}^{*}$ in step 4 of procedure II for $\beta^{\prime}$ number of periods (where $S_{\min }$ is the minimum active storage requirement that may be violated $\beta$ fraction of the time; $\beta^{\prime}=$ rnd $\left.\{Y S \beta\}\right)$ should achieve that.

Any algorithm that calculates $K_{o}^{*}$ directly obviates the need to include the $2 T$ constraints ( $T$ constraints for storage continuity and $T$ for minimum storage) and the $T$ variables (corresponding to the storage in each time period) in the reservoir capacity optimization problem. It is this large number of constraints and variables that imposes significant limits on the size of the LP problem that can be solved practically (and forces analysts to look for alternatives to the "complete" model like the "critical sequence of years" model or the "approximate within-year and over-year separated sequences" model [Loucks et al., 1981, pp. 343-348]). Conversely, our algorithms, with their very small computational requirements and their "self-contained" form, could be used for solving models with a very large number of periods and even in multireservoir models.

Further, since the remaining constraints and variables are much fewer in number (typically involving only the withinyear releases, the active storage capacity, and related constraints), it is possible to solve nonlinear problems using direct-search methods for constrained nonlinear optimization, with the storage capacity being calculated at each point in the search using one of our algorithms. For example, for a singlereservoir 100-year and 12-month model, if the objective function is nonlinear (say, because of a nonlinearly varying dam cost), the LP approach requires the use of a piecewise linear approximation and integer programming; the problem would then be of more than 1200 variables and more than 2400 constraints. Instead, one could use algorithm I and pose the problem as one in which only the monthly releases are the decision variables. This would reduce the problem to a 12variable (and maybe 12 bounds) problem; existing computerized algorithms can solve nonlinear constrained optimization problems of this size quite rapidly. (See Lele [1986, chap. 3] for such an application.) Since the algorithm calculates the values of $S_{t}$ for all $t$, it could be used even in the case of a hydropower generation model wherein the objective function is nonlinear because the generation head is a function of the storage level.

When specifying the minimum reliability norm for water resources projects, decision-making bodies often also specify the extent of shortfall that may be allowed in any failure year. For instance, the Planning Commission in India specifies that the reliability of hydropower and irrigation projects may not be less than $90 \%$ and $75 \%$, respectively, and the shortfall in any failure year may not be more than $15-20 \%$ (Y. D. Pendse, Central Water Commission, New Delhi, personal communication, 1985). The LP formulation uses chance-constrained models for such problems, which require the estimation of the cumulative distribution functions for the seasonal inflows; they also usually do not specify the extent of shortfall. Rather than go through these complex calculations, planners often prefer to design approximately. In doing so, they err on the side of overdesigning, i.e., for excessively high reliability and low shortfall. Calculations show that the difference between the storage capacity requirements at, say, $99 \%$ and $90 \%$ reliability could be very significant; for the inflow sequence mentioned above, it was $30 \%$. Similarly, exact consideration of shortfall could also affect the storage capacity calculation significantly. Overdesigning could turn out to be quite costly, especially when one considers the environmental impacts of land submergence. Procedure II provides a simple, fast, and accurate method for recalculating the capacity and thus for examining the tradeoffs between reliability and economic and/or environmental costs.

Acknowledgments. The author wishes to thank S. Vedula of the Indian Institute of Science, Bangalore, and Daniel P. Loucks of Cornell University, Ithaca, for their encouragement. The prompt and valuable comments of the anonymous referee are also gratefully acknowledged.

Lele, S. M., An optimization model for macro-hydeł projects incorporating energy/economic costs of submergence, M.S. thesis, 160 pp., Indian Inst. of Sci., Bangalore, 1986.

Loucks, D. P., Surface water quantity management models, in Systems Approach to Water Management, edited by A. K. Biswas, pp. 167-170, MoGraw-Hill, New York, 1976.

Loucks, D. P., J. R. Stedinger, and D. A. Haith, Water Resources Systems Planning and Analysis, chaps. 5 and 7, Prentice-Hall, Englewood Cliffs, N. J., 1981.

Murray, M., and $\mathbf{K}$. Weissbeck, Computer program for river hydro development, Intl. Water Power Dam Constr., 30(3), 30-40, 1980.

Stedinger, J. R., The performance of LDR models for preliminary design and reservoir operation, Water Resour. Res., 20(2), 215-224, 1984. 
Tejada-Guibert, J. A., Algoritmos para la determinacion de capacidades de embalse (Algorithms for the determination of reservoir capacities), paper presented at the Eighth Latin-American Congress on Hydraulics, Quito, Ecuador, 1978.

Thomas, H. A., Jr., and M. B. Fiering, Operations Research in Water Quality Management, pp. 1-17, Harvard Water Resources Group, Cambridge, Mass., 1963.
S. M. Lele, Energy and Resources Group, Building T-4, University of California, Berkeley, CA 94720.

(Received May 7, 1987;

revised July 13, 1987;

accepted July 16, 1987.) 